\title{
An Edge-based Galerkin Formulation for Thermal Non-Equilibrium Flows
}

\author{
Song Gao ${ }^{1}$, Wagdi G. Habashi ${ }^{2}$ \\ NSERC-Lockheed Martin-Bell Helicopter Industrial Research Chair \\ for Multi-physics Analysis and Design of Aerospace Systems \\ CFD Laboratory, McGill University, Montreal, Quebec, H3A 2S6, Canada \\ Dario Isola ${ }^{3}$ and Guido S. Baruzzi ${ }^{4}$ \\ ANSYS, Inc., Montreal, QC, H3A 3G4, Canada \\ Marco Fossati ${ }^{5}$ \\ University of Strathclyde, Glasgow, G1 1XJ, UK \\ Luke Uribarri ${ }^{6}$ \\ Lockheed Martin Corporation, Bethesda, MD, 20817, USA
}

\begin{abstract}
A parallel finite element solver is developed for hypersonic flows with frozen chemistry. The finite element formulation is edge-based with flow stabilization via a Roe scheme. The flow solver is loosely-coupled with a two-temperature thermal non-equilibrium solver. Numerical experiments are performed to assess the accuracy and efficiency of the proposed approach.
\end{abstract}

\section{Introduction}

$\mathrm{T}$ HE aviation industry is involved in studies of high-speed non-equilibrium flows. Strong shock waves, chemical reactions, thermal non-equilibrium and the potential presence of free electrons that may induce magnetogasdynamic effects make the physics of such flows complex [1,2]. To achieve reliable design of high-speed civil transport, the accurate prediction of shear stresses, heat fluxes and temperature distributions over the surface of the vehicle are essential. Experimental tests $[3,4]$, together with flexible and reliable computational fluid dynamics (CFD) tools, are critical in understanding such high-speed flows.

${ }^{1}$ Doctoral candidate, CFD Laboratory, Department of Mechanical Engineering, 688 Sherbrooke St. West, AIAA Member.

2 Professor and Director CFD Laboratory, Chairholder, Department of Mechanical Engineering, 688 Sherbrooke St. West, AIAA Fellow.

${ }^{3}$ Senior Software Developer, ANSYS Canada, 1000 Sherbrooke St. West, AIAA Member.

${ }^{4}$ Technical Fellow, ANSYS Canada, 1000 Sherbrooke St. West, AIAA Member.

${ }^{5}$ Lecturer and Chancellor Fellow, Department of Mechanical and Aerospace Engineering, 75 Montrose Street, AIAA Member.

${ }^{6}$ Staff Aeronautical Engineer, Advanced Technology Laboratories, AIAA Member.

This is a peer-reviewed, accepted author manuscript of the following article: Gao, S., Habashi, W. G., Isola, D., Baruzzi, G. S., Fossati, M., \& Uribarri, L. (2018). An edge-based Galerkin formulation for thermal non-equilibrium flows. In AIAA Aerospace Sciences Meeting [AIAA 2018-0064] American Institute of Aeronautics and Astronautics Inc, AIAA. https://doi.org/10.2514/6.2018-0064 
Several numerical approaches for high-speed flows have been developed based on the finite volume (FV) method [5-7] and the finite element (FE) method [8,9]. FE methods have favorable properties such as rigorous formulation of boundary conditions, more accurate derivatives on surfaces and superior robustness on stretched grids. In particular, the FE method implemented in this work uses an edge-based formulation that introduces favorable numerical properties, such as the straightforward application of upwind numerical schemes at cell interfaces, an efficient (cost and memory-wise) framework to deal with complex meshes (structured, unstructured and hybrid) within a unique data structure and a more computationally efficient assembly compared to elementbased ones $[10,11]$.

The studies of high-speed flows involve solving a system of conservation equations that model non-equilibrium. The degree of thermal non-equilibrium is typically measured by a suitable Damköhler number, expressed as the ratio between the characteristic time for a fluid element to travel a characteristic length and the characteristic time for the energy modes to reach equilibrium [7]. When the Damköhler number is of the order of unity, the flow is in thermal non-equilibrium and a local equilibrium approach, where all energy modes are in equilibrium at one temperature, is not suitable to characterize the aerothermodynamic processes. To properly address the effects of thermal nonequilibrium, the three-temperature model $[12,13]$ introduces a translational-rotational temperature to characterize the translational and rotational kinetic energies of the particles, a vibrational temperature to account for the energy associated with the deformation of polyatomic molecules with multiple atoms and chemical bonds, and an electronic-electron temperature due to the presence of free electrons and electronic excitation of the species constituting the gas. Although this approach is often presented in the literature, it does not yield accurate results and is rarely used in high-speed codes [7]. The thermal non-equilibrium model used in this work is Park's twotemperature model, which further assumes the vibrational temperature and electronic-electron temperature to have the same value [14]. This model simplifies computational complexity, and provides good results for aerodynamic coefficients and convective heat transfer rates [13].

The present work introduces a loosely coupled edge-based FE solver for the modeling and simulation of highMach flows in thermal non-equilibrium. The computational framework will be discussed and validated without considering the presence of chemical reactions at this stage. This is justified by the fact that in high-enthalpy flows, thermal non-equilibrium is often relevant even before any chemical reaction is activated and therefore this approach provides a meaningful extension of local / quasi-local equilibrium approaches to address high-Mach flows. A finite element edge-based formulation [10] is used to reduce the cost of the assembly and to enable the application of Roe scheme [15] for its superior accuracy, stability and efficiency in various flow regimes. Second order accuracy in space is achieved via the Monotonic Upstream-Centered Scheme for Conservation Laws (MUSCL) [16]. The methodology introduced is intended to serve as a framework for the eventual simulation of flows with more complex physics and thermodynamics properties.

The manuscript is organized as follows: Section II presents the stable edge-based FE scheme. Section III presents numerical results and Section IV provides conclusions and future work.

\section{Edge-based formulation of the FE scheme}

The governing equations of a single-species steady-state viscous flow in thermal non-equilibrium can be written as [12]

$$
\nabla \cdot\left(\mathbf{F}^{A}(Q)-\mathbf{F}^{V}(Q, \nabla Q)-\mathbf{F}^{T}(Q, \nabla Q)\right)=S(Q)
$$

where $Q$ is the vector of conserved variables, $Q=\left[\rho, \rho \boldsymbol{V}, \rho E, \rho E_{v e}\right], \rho$ is the density, $\boldsymbol{V}$ is the velocity vector, $E$ is the total energy per unit mass, $E_{v e}$ is the total vibrational-electronic energy per unit mass, $\mathbf{F}^{A}, \mathbf{F}^{V}, \mathbf{F}^{T}$ are, respectively, the inviscid, viscous and thermal flux vectors. $\mathbf{F}^{T}=\left[0, \mathbf{0}, k_{t r} \Delta T+k_{v e} \Delta T_{v e}, k_{v e} \Delta T_{v e}\right]$, where $k_{t r}$ and $k_{v e}$ are, respectively, the heat conduction coefficient for translational-rotational energy and vibrational-electronic energy, $\Delta T$ and $\Delta T_{v e}$ are the Laplacian of translational-rotational temperature and vibrational-electronic temperature, $S=\left[0, \mathbf{0}, 0, S_{t-v}\right] . S_{t-v}$ is the energy relaxation between vibrational and translational energies, modeled by the Landau-Teller theory [12], i.e.

$$
S_{t-v}=\rho \frac{E_{v}(T)-E_{v}\left(T_{v e}\right)}{\tau}
$$


where $E_{v}$ is the vibrational energy per unit mass, $T$ is the translational-rotational temperature and $\tau$ is the relaxation time modeled by the Millikan-White formula [17] and the Park's correction [14].

The weak-Galerkin method is used to obtain a FE representation of Eq. (1) [11]

$$
\sum_{e \in E_{i}} \int_{Y_{e}} \nabla W_{i} \cdot\left(\mathbf{F}^{A}-\mathbf{F}^{V}-\mathbf{F}^{T}\right) d \forall+\sum_{e \in F_{i}} \int_{A} W_{i} \mathbf{n} \cdot\left(\mathbf{F}^{A}-\mathbf{F}^{V}-\mathbf{F}^{T}\right) d A=\sum_{e \in E_{i}} \int_{H_{e}} W_{i} S d V
$$

where $W_{i}$ is the linear Lagrangian test function and $E_{i}$ and $F_{i}$ are the set of elements/faces sharing the $i$-th vertex. The second term of equation (3) is the boundary term. Since in 3D, the boundary elements are much fewer than the volume elements, this term is evaluated in standard FE schemes by simply looping over the faces of the elements that belong to the boundary. For simplicity, the contribution from the boundary conditions will be ignored from this point on. The first and last terms of the equation (3) can be assembled in an edge-based fashion as [11]

$$
\sum_{j \in K_{i}} \boldsymbol{\eta}_{i j} \cdot \frac{\mathbf{F}_{j}^{A}+\mathbf{F}_{i}^{A}}{2}-\sum_{j \in K_{i}} \boldsymbol{\chi}_{i j} \cdot \frac{\mathbf{F}_{j}^{A}-\mathbf{F}_{i}^{A}}{2}+\sum_{e \in E_{i} Y_{e}} \int_{i} W_{i} \cdot\left(\mathbf{F}^{V}+\mathbf{F}^{T}\right) d \forall=-L_{i} S_{i}
$$

where $K_{\mathrm{i}}$ is the set of nodes connected to the $i$-th node and the lumped mass matrix is introduced as

$$
L_{i}=\sum_{j \in K_{i}} \sum_{e \in E_{i}} \int_{V_{e}} W_{i} W_{j} d \forall
$$

The edge coefficient vectors are defined as

$$
\boldsymbol{\eta}_{i j}=\sum_{e \in E_{i}}\left(\int_{V_{e}} W_{i} \nabla W_{j} d \forall-\int_{V_{e}} W_{j} \nabla W_{i} d \forall\right) \quad \boldsymbol{\chi}_{i j}=\sum_{e \in E_{i}} \int_{A} W_{i} W_{j} \mathbf{n} d A
$$

where $\chi_{i j}$ is a boundary contribution different from zero only for boundary faces.

The above FE discretization is known to be unstable for advection-dominated flows. The Roe scheme is used to provide a stabilized numerical formulation of the inviscid fluxes. The vector of inviscid fluxes $\mathbf{F}^{A}$ of Eq. (4) is replaced with the numerical approximation $\mathbf{F}^{\text {Roe }}$. The inviscid fluxes used in Eq. (4) are replaced by their numerical counterpart evaluated at the edge midpoint

$$
\sum_{j \in K_{i}} \boldsymbol{\eta}_{i j} \cdot \mathbf{F}^{R o e}\left(Q_{i}, Q_{j}\right)-\sum_{j \in K_{i}} \boldsymbol{\chi}_{i j} \cdot \frac{\mathbf{F}_{j}^{A}-\mathbf{F}_{i}^{A}}{2}+\sum_{e \in E_{i}} \int_{V_{e}} \nabla W_{i} \cdot\left(\mathbf{F}^{V}+\mathbf{F}^{T}\right) d \forall=-L_{i} S_{i}
$$

The flux $\mathbf{F}^{R o e}$ in Eq. (5) is a non-linear function of the nodal variables of the edge. The second term in Eq. (5) is a "correction" factor that is only nonzero at the boundary edges, since it is proportional to the difference of the fluxes. Following [10], the natural inviscid flux is left unchanged and no additional dissipation is introduced.

The resulting scheme is first-order in space and is made second-order by introducing the MUSCL approach. In this work, the van Albada slope limiter [18] is used. The viscous and thermal fluxes are discretized with the standard continuous Galerkin approximation and assembled in an edge-based fashion, naturally resulting in a second-order representation. The viscosity coefficient is computed using NIST polynomials [19] and the heat conduction coefficients are from Eucken's relation [6]. A loosely-coupled strategy is used, that splits Eq. (1) into two systems. The conservation of mass, momentum and total energy are combined as the first system, and the conservation of vibration-electronic energy is the second one. Each system of equations is solved independently with information exchange through the variables $T, \boldsymbol{V}, \rho$ and $T_{v e}$, as depicted in Figure 1. 


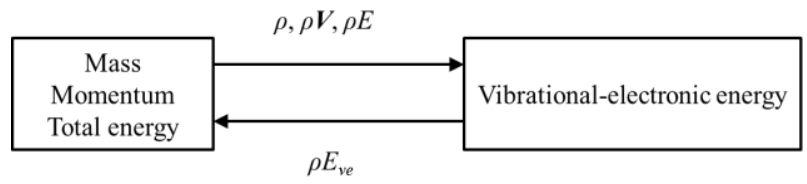

Figure 1. Diagram of the coupling between Navier-Stokes and thermal non-equilibrium solver

\section{Numerical results}

Three test cases are presented. The first one validates the source term in the vibration-electronic energy equation that models the energy exchange between the translational and vibrational modes. The second test case consists of Mach 20 flow past a cylinder in 2D. The third one is a Mach 11.3 flow past a blunt cone in 3D. These cases are used to examine the accuracy and performance of the proposed formulation. In all cases, chemical reactions are not considered.

\section{A. Zero-dimensional cube}

To validate the numerical formulation of the source term in vibration-electronic energy equation, the NavierStokes solver is turned off. The test case is a cube filled with Oxygen at rest. Consequently, the convection term in the vibration-electronic energy solver vanishes, and each grid node is decoupled from others. Initially, the pressure is $0.063 \mathrm{~atm}$, the translational-rotational temperatures are $300 \mathrm{~K}$, and the vibrational-electronic temperatures is $20,000 \mathrm{~K}$. A constant time step of $1 \times 10^{-10} \mathrm{~s}$ is used.

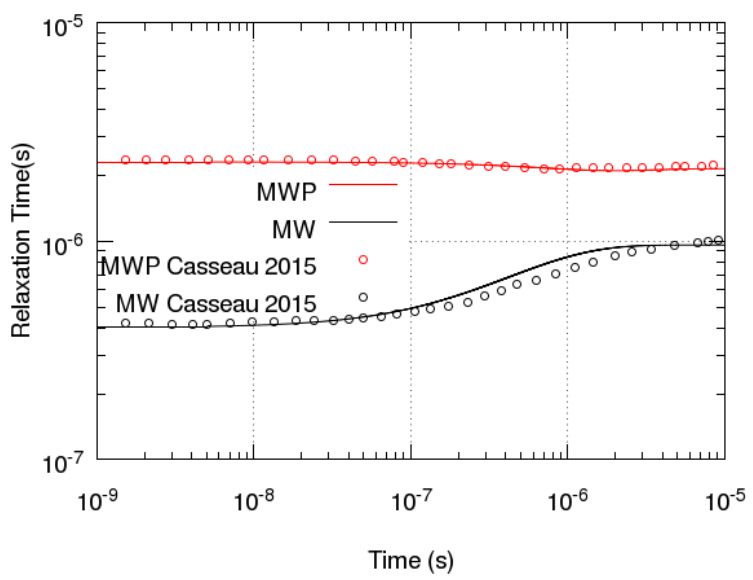

Figure 2. Zero-dimensional cube: Translational-vibrational relaxation time versus physical time

Figure 2 plots the translational-vibrational relaxation time versus physical time. In the legend, MW corresponds to the relaxation time computed by Millikan-White formula and MWP stands for the relaxation time computed by Millikan-White's formula with Park's correction. Millikan-White's formula is a semi-empirical curve valid in the temperature range from $300 \mathrm{~K}$ to $8000 \mathrm{~K}$. At higher temperature, it is known that the Millikan-White formula underpredicts the relaxation time. Park corrected this by adding an extra term that more accurate estimates collision cross sections. In Figure 3 (left), the translational-rotational (T-R) temperatures and the vibrational-electronic (V-E) temperatures are plotted against the reference, and it can be seen that the T-R and V-E temperatures eventually reach equilibrium. MW reaches equilibrium earlier than MWP, indicating that Park's correction increases the relaxation time at higher temperatures. Figure 3 (right) plots the pressure distributions versus time. The solid lines are results from this work and the circle points are reference results from [20]. Good agreement is found for all the results. 

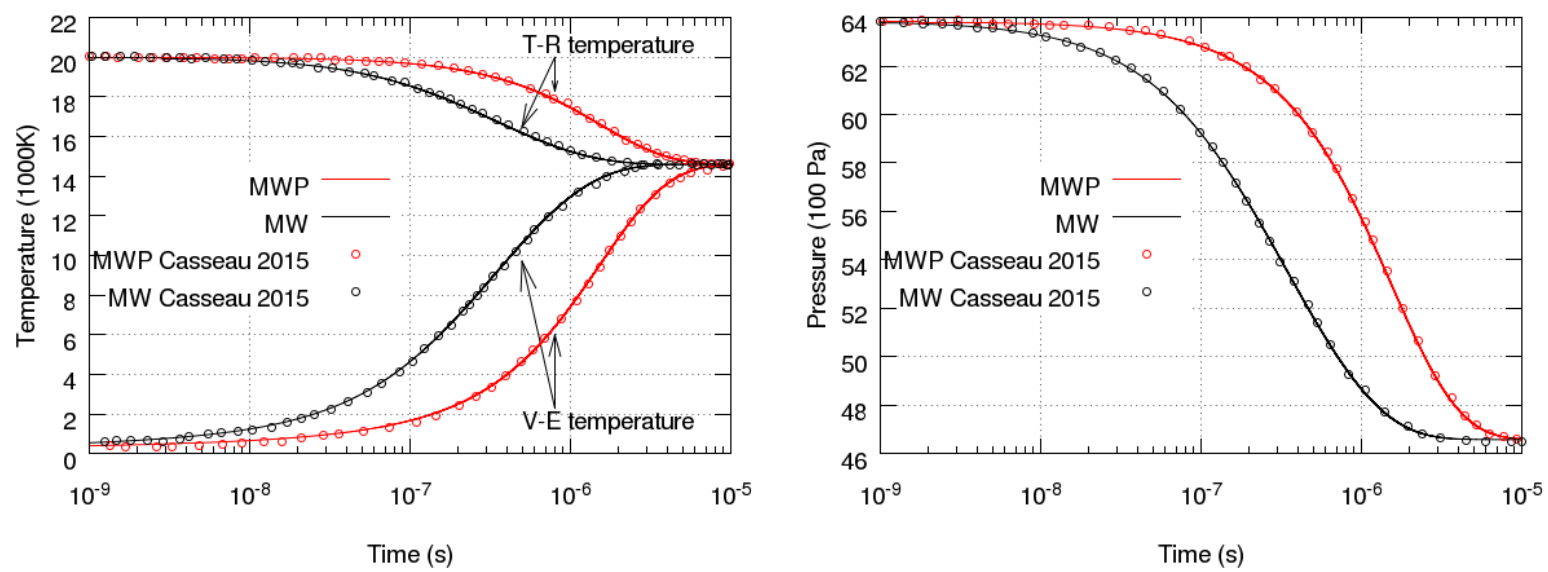

Figure 3. Zero-dimensional cube: translational-rotational temperature and vibrational-electronic temperature versus physical time (left). Pressure distribution versus physical time (right).

\section{B. Mach 20 flow past a cylinder}

The second test case is a Mach 20 laminar flow of Nitrogen past a cylinder in 2D. The radius is $1 \mathrm{~m}$. The Reynolds number based on cylinder radius is 5,913. A 2D structured mesh is shown in Figure 4, consisting of 78,400 nodes and 78,204 elements. The height of the first layer of elements near the wall is $2 \mu \mathrm{m}$. The solution is obtained with the Roe scheme and extended to second order by the van Albada slope limiter. The initial conditions are: Mach number 20, velocity $6047 \mathrm{~m} / \mathrm{s}$, pressure $0.89 \mathrm{~Pa}$, both freestream translational-rotational and vibrationalelectronic temperatures are $220 \mathrm{~K}$. The two temperatures on the wall are set to $1,000 \mathrm{~K}$. The far-field Knudsen number $(\mathrm{Kn})$ is $5.1 \times 10^{-3}$.
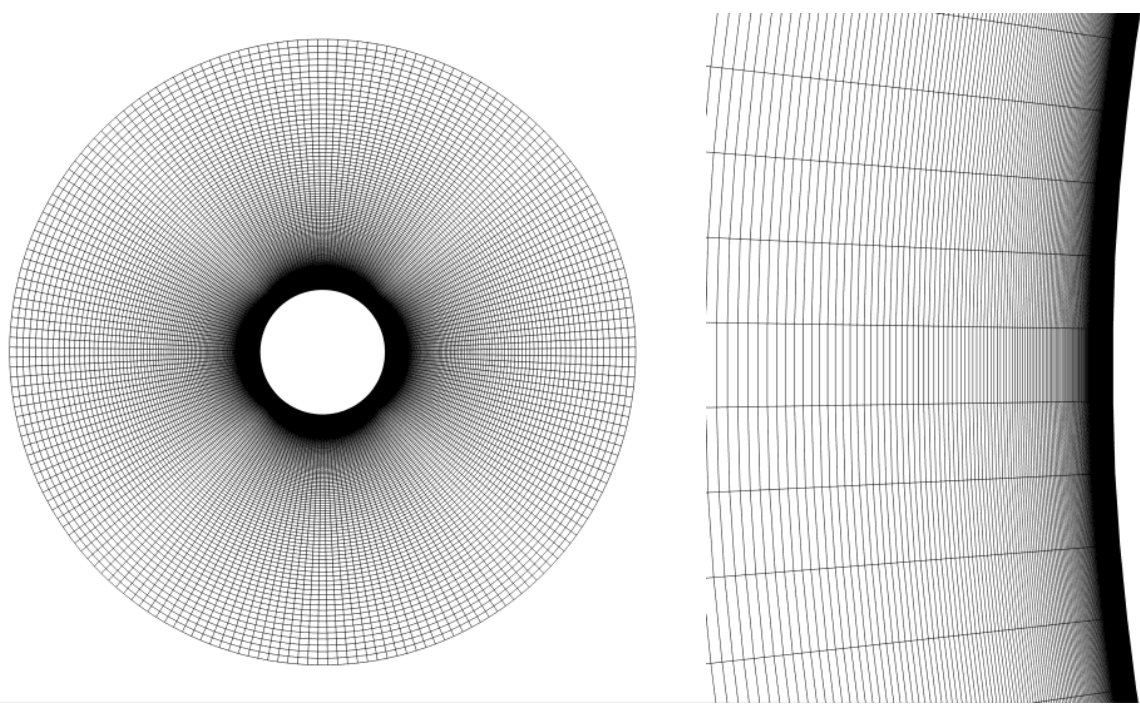

Figure 4. Mach 20 flow past a cylinder: mesh (left) and mesh near the wall (right) 

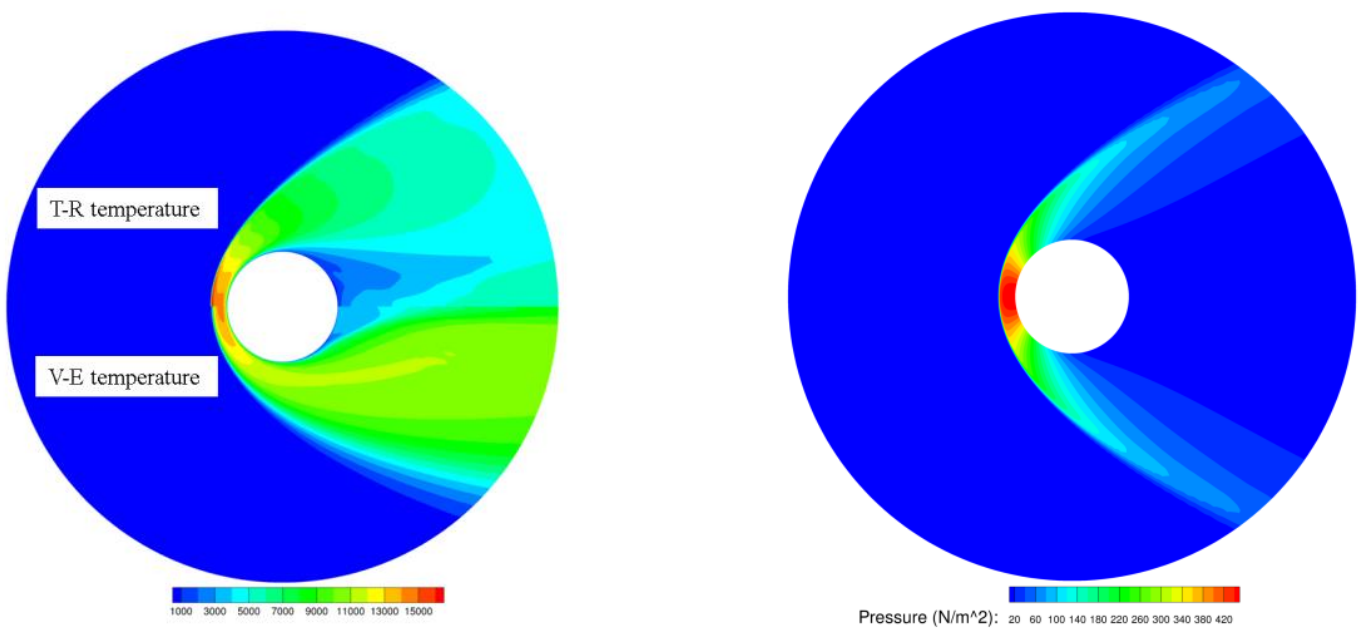

Figure 5. Mach 20 flow past a cylinder: Translational-rotational and vibrational-electronic temperature contours (left) and pressure contour (right).

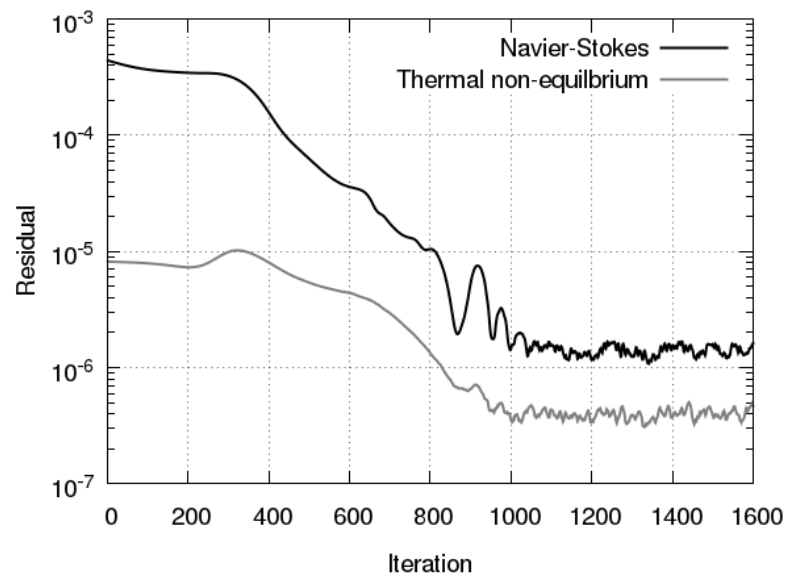

Figure 6. Mach 20 flow past a cylinder: convergence curves 

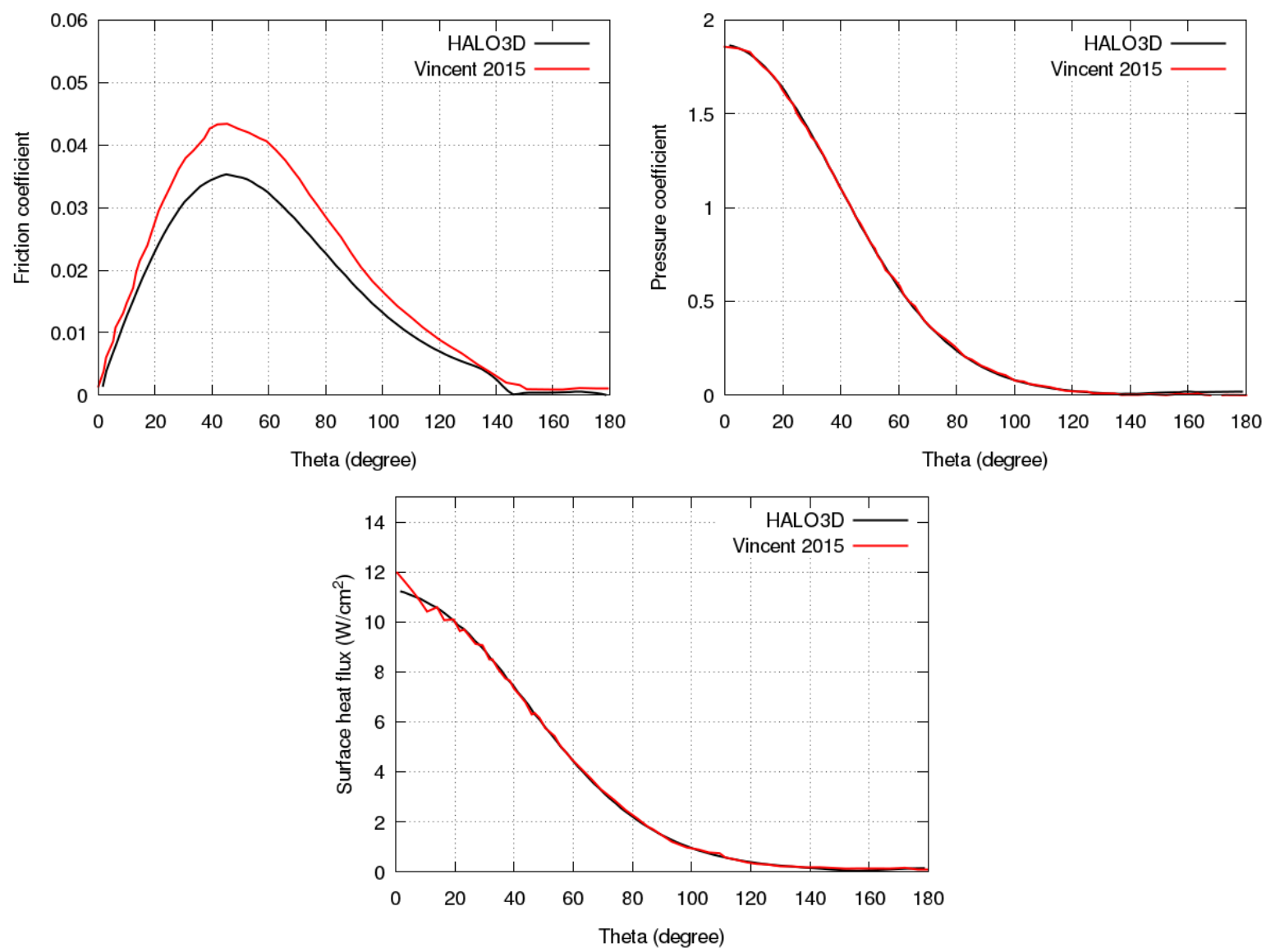

Figure 7. Mach 20 flow past a cylinder: skin friction coefficient, pressure coefficient and surface heat flux on the wall.

Figure 5 plots the translational-rotational and vibrational-electronic temperature contours on the left, and pressure contours on the right. Figure 6 plots the convergence curves for Navier-Stokes and thermal non-equilibrium solver. The Navier-Stokes solver achieves roughly three orders of magnitude reduction in the residual norm, with both solvers stalling after 1,000 Newton iterations. Figure 7 plots the skin friction coefficient, pressure coefficient and heat fluxes on the wall. Figure 8 plots the translational-rotational temperature, vibrational-electronic temperature, density and Mach number profiles along the stagnation line. The CFD results with the no-slip boundary condition (labelled as HALO3D) are compared with Casseau's results (labelled as Vincent 2015) where the Smoluchowski temperature jump condition and the Maxwell velocity slip jump boundary conditions are employed [6]. Since the far-field $\mathrm{Kn}$ number is still in the continuum regime, minor differences are expected between the profiles obtained from the two boundary conditions. With the exception of the friction coefficient on the wall, which is under-predicted, the comparisons of other quantities are in good agreement. 

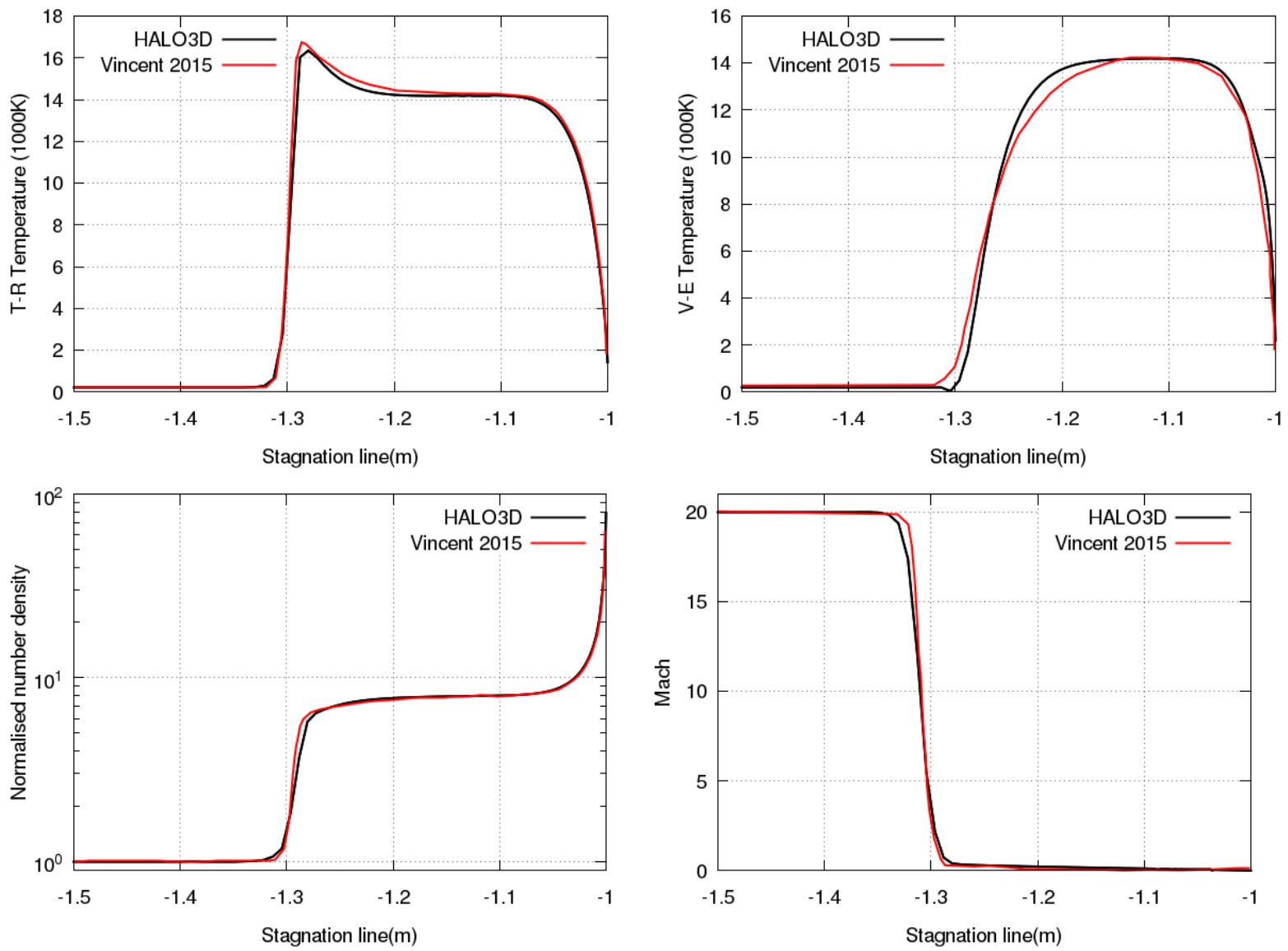

Figure 8. Mach 20 flow past a cylinder: translational-rotational temperature, vibrational-electronic temperature, density and Mach number along the stagnation line.

\section{Mach 11.3 flow past a blunt cone}

The third test case is a Mach 11.3 laminar flow of Nitrogen past a blunt cone in 3D. The nose radius is $6.35 \mathrm{~mm}$. The flat plane is $5 \mathrm{~cm}$ long in the streamwise direction and has a 25-degree angle with the stream direction. The Reynolds number based on the length of the blunt cone is 8,284. A 3D structured mesh is shown in Figure 9, consisting of 8,611,400 nodes and 8,568,144 elements. The height of the first layer of elements near the wall is 2 $\mu \mathrm{m}$. The solution is obtained using the Roe scheme and extended to second order by the van Albada slope limiter. The initial conditions are: Mach number 11.3, velocity $2764.5 \mathrm{~m} / \mathrm{s}$, pressure $21.9 \mathrm{~Pa}$, freestream translationalrotational and vibrational-electronic temperatures are $144.4 \mathrm{~K}$. Both temperatures on the wall are $297.2 \mathrm{~K}$. The farfield $\mathrm{Kn}$ number is $2.05 \times 10^{-3}$. 


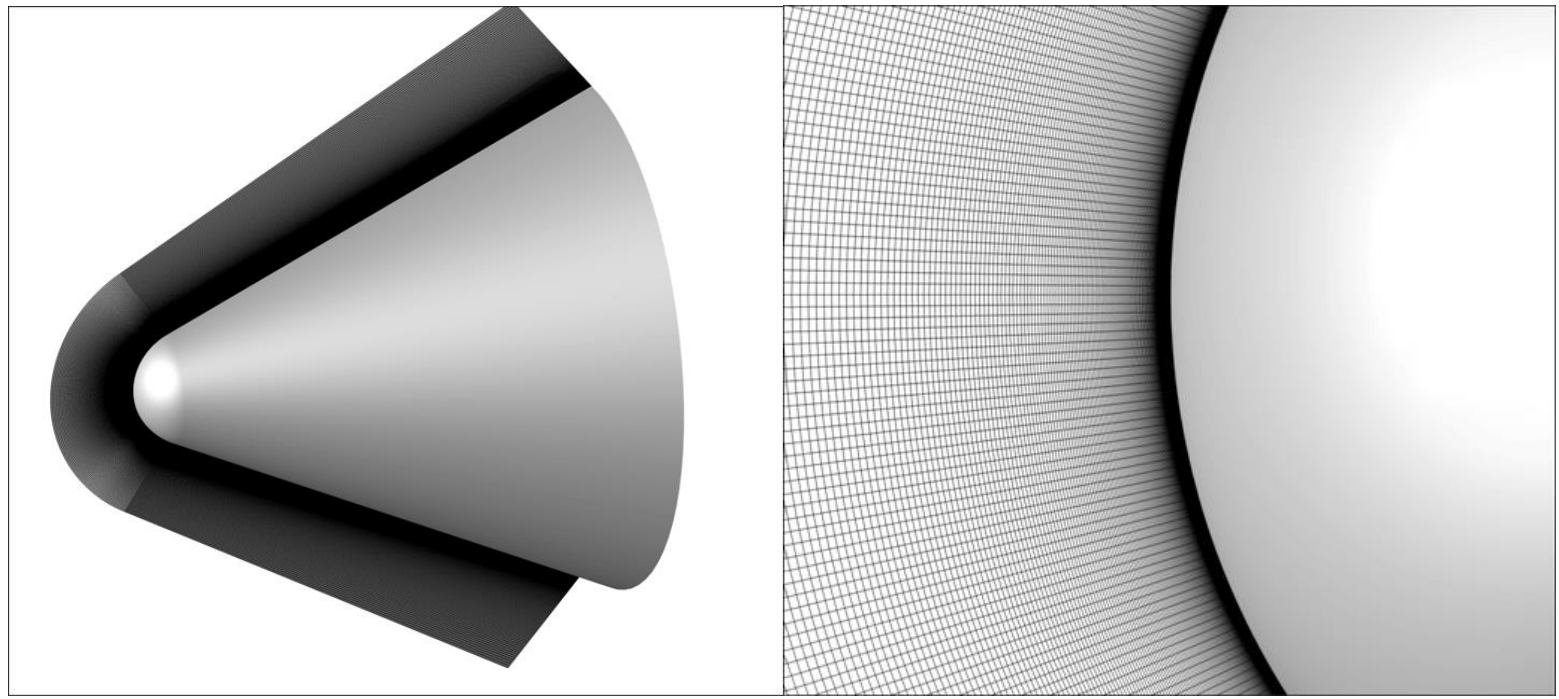

Figure 9. Mach 11.3 flow past a blunt cone: mesh (left) and mesh near the wall (right)
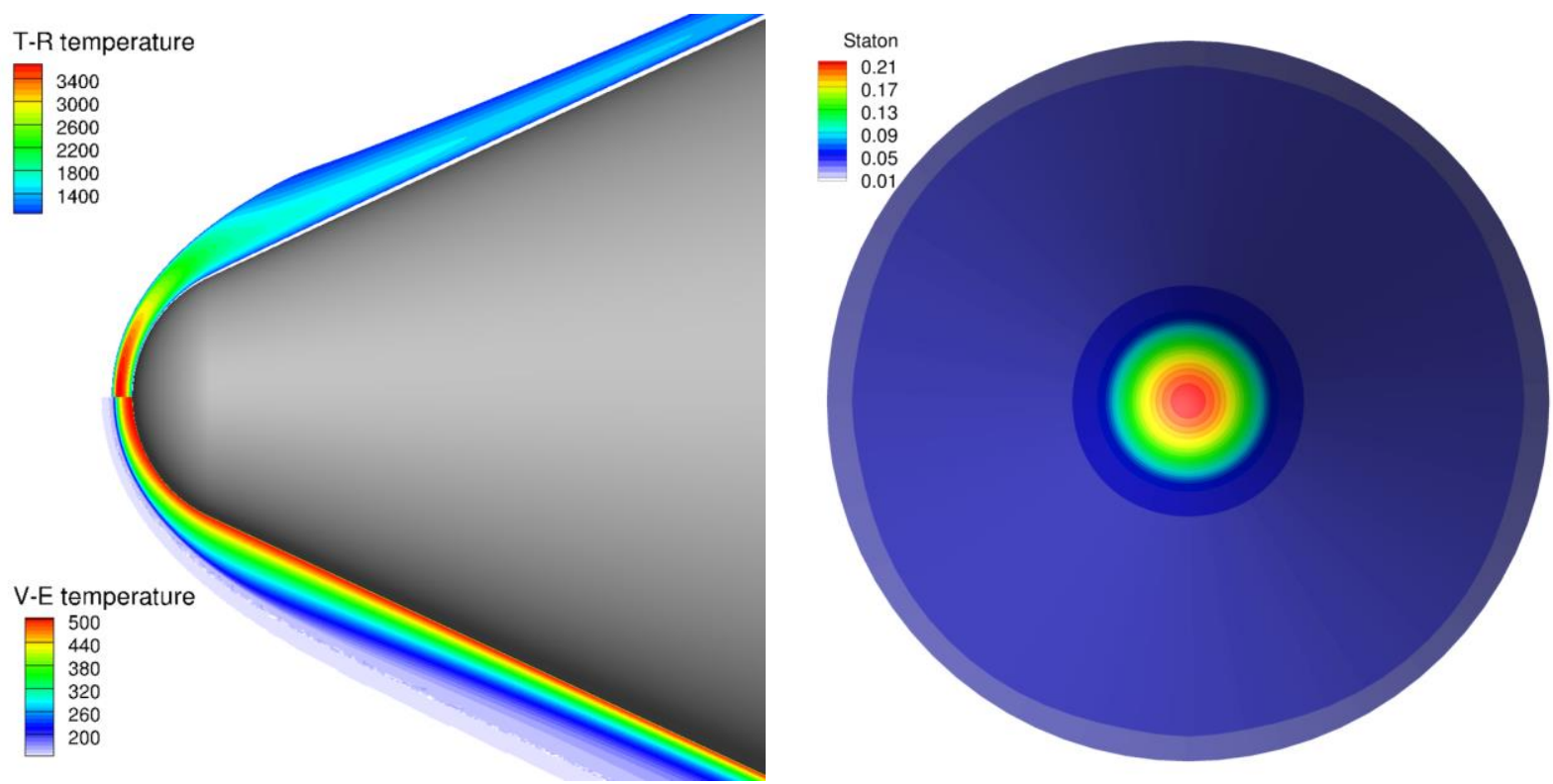

Figure 10. Mach 11.3 flow past a blunt cone: Translational-rotational and vibrational-electronic temperature contours (left) and Stanton number contour (right).

Figure 10 plots the translational-rotational and vibrational-electronic temperature contours on the left, and Stanton number contours on the right. Figure 11 plots the convergence curves for Navier-Stokes and thermal nonequilibrium solver. The Navier-Stokes solver achieves roughly four orders of magnitude reduction in the residual norm, with both solvers stalling after 3,000 Newton iterations, possibly due to the loosely coupled strategy. Figure 12 plots the skin friction coefficient, pressure coefficient and Stanton numbers on the wall. The CFD results with the no-slip boundary condition are compared with Casseau's results where the Smoluchowski temperature jump condition and the Maxwell velocity slip jump boundary conditions are employed [6]. As in the cylinder test case, the far-field Kn number is still in the continuum regime and little difference is expected to be found between the profiles obtained from the two boundary conditions. Figure 13 plots the translational-rotational temperature, vibrationalelectronic temperature, density and velocity profiles along the stagnation line. The translational-rotational temperature profile is slightly under-predicted compared to Casseau's profile and the vibrational-electronic temperature profile is slightly different from Casseau's profile. Further investigation is required on this issue and 
this author is being contacted. The agreement of density and velocity profiles along the stagnation line is satisfactory.

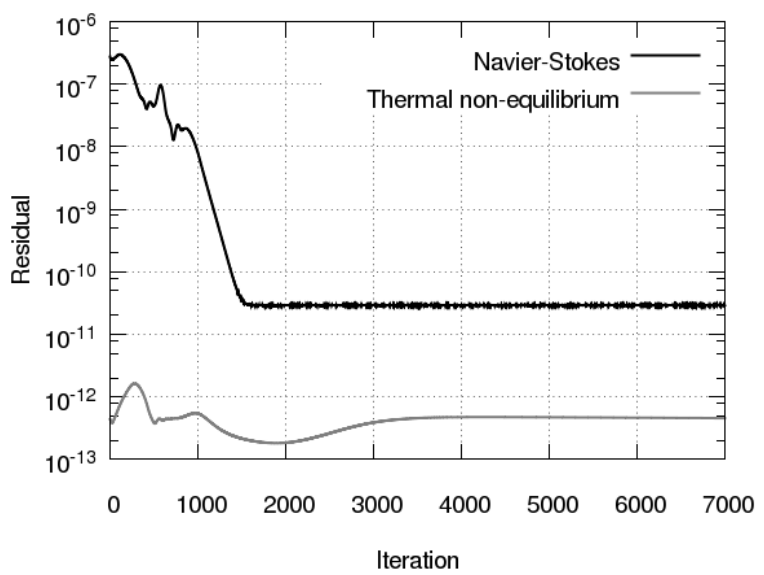

Figure 11. Mach 11.3 flow past a blunt cone: convergence curves
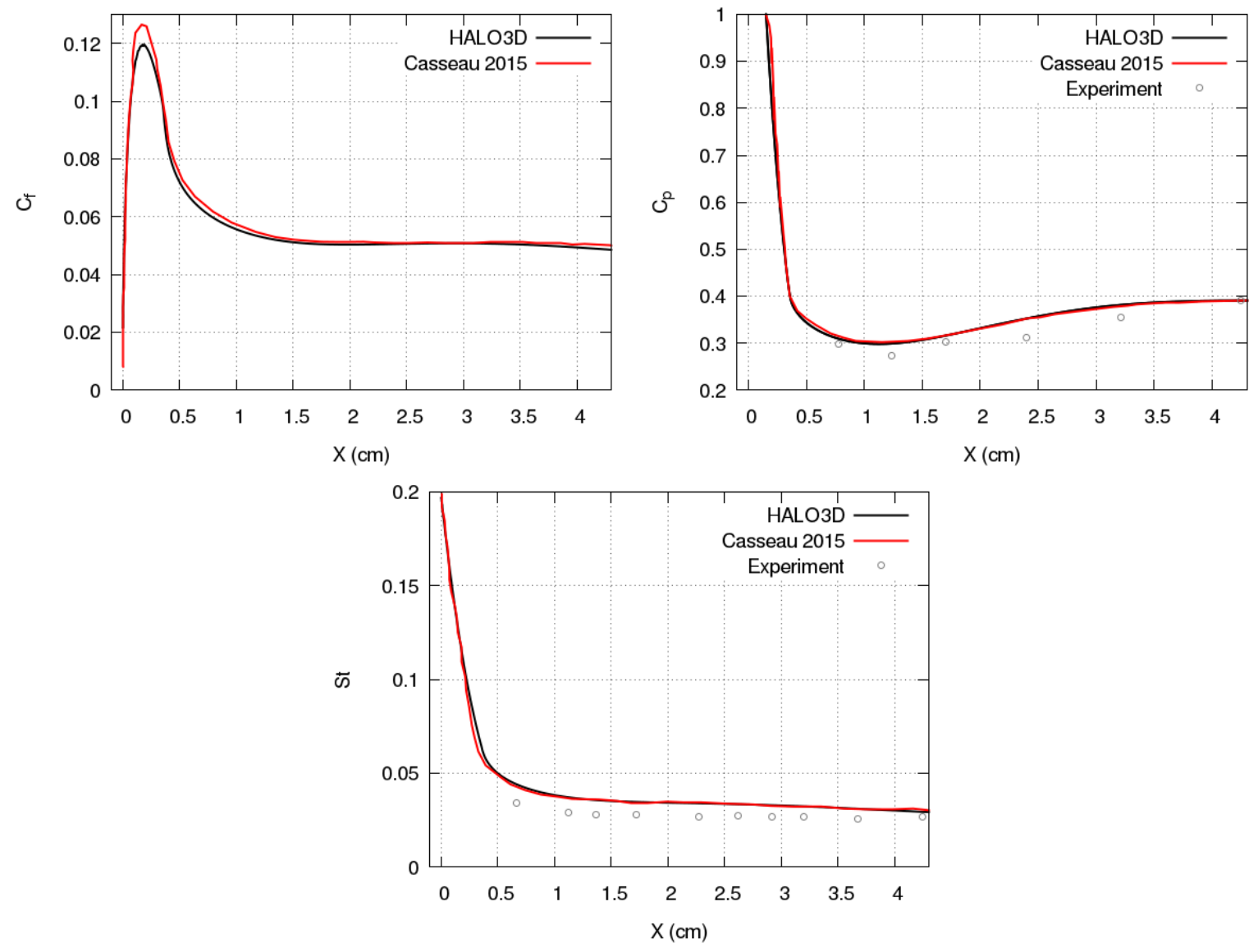

Figure 12. Mach 11.3 flow past a blunt cone: skin friction coefficient, pressure coefficient and Stanton number on the wall. 

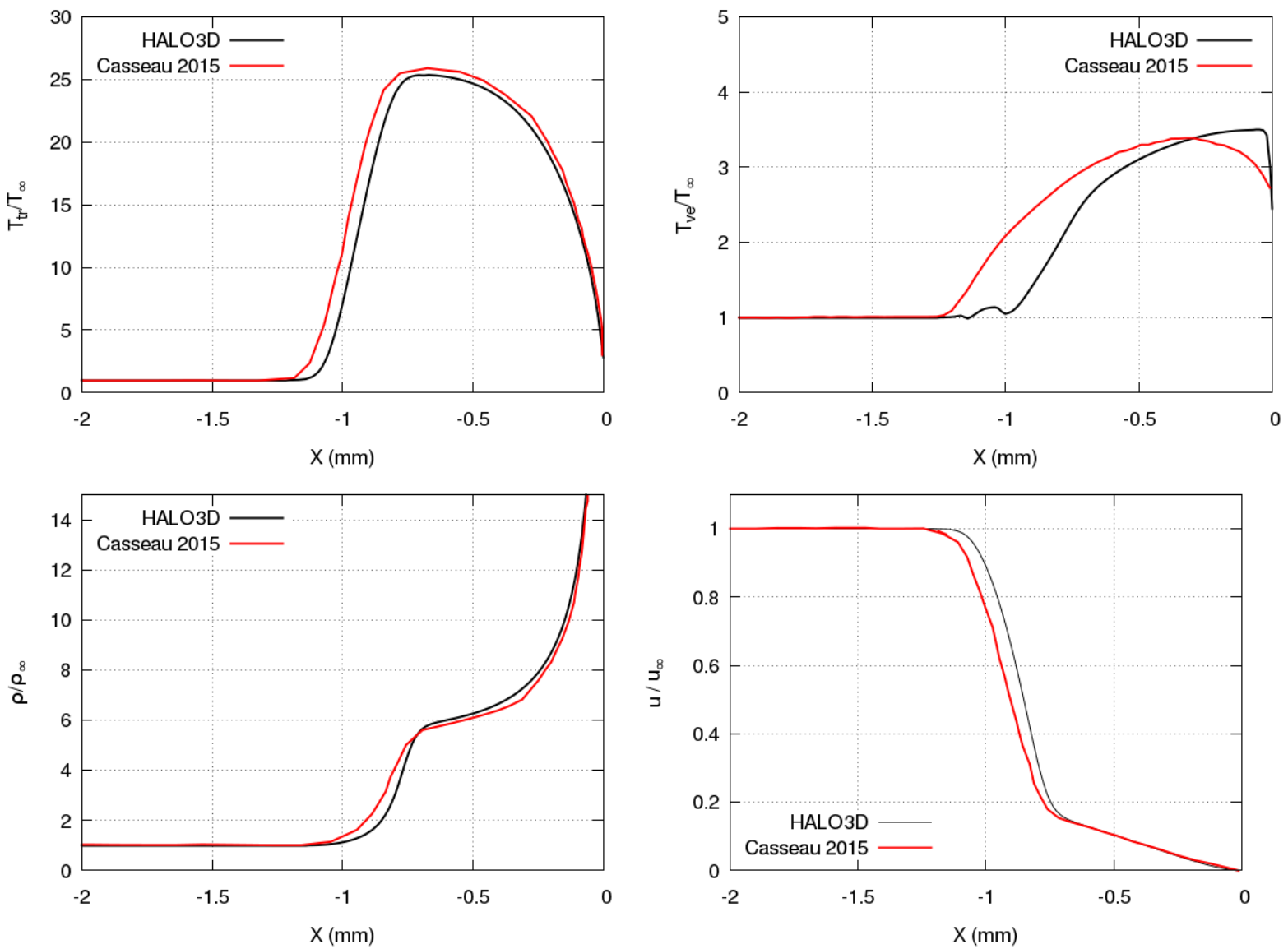

Figure 13. Mach 11.3 flow past a blunt cone: translational-rotational temperature, vibrational-electronic temperature, density and velocity along the stagnation line.

\section{Conclusions}

The present work is part of a large and sustained effort to develop an edge-based FEM all speeds compressible turbulent flow solver, suitable for hypersonic flows. The present paper addresses a robust and accurate loosely coupled solver for high-Mach thermal non-equilibrium flows with frozen chemistry. The two-temperature model is used to model the thermal non-equilibrium. The edge-based strategy yields a stable FE formulation with the numerical fluxes computed by the Roe scheme. Various zero, two and three-dimensional numerical results are presented. These results are compared with references and good agreement is found in all test cases.

\section{Acknowledgments}

The authors acknowledge the financial support of the NSERC-Bell Helicopter-Lockheed Martin Industrial Research Chair at the McGill CFD Lab, and through Lockheed Martin grant G238199 to ANSYS. We are also grateful to Compute Canada and CLUMEQ for access to their computational resources that facilitated this work.

\section{References}

1. Barbante, P.F. and Magin, T.E., Fundamentals of Hypersonic Flight-properties of High Temperature Gases. RTO AVT Lecture Series on Critical Technologies for Hypersonic Vehicle Development, 2004: pp. 5.1-5.50.

2. Moses, P.L., Rausch, V.L., Nguyen, L.T., and Hill, J.R., NASA Hypersonic Flight Demonstrators-Overview, Status, and Future Plans. Acta Astronautica, 2004. 55(3): pp. 619-630. 
3. Eggers, T., Stamminger, A., Hörschgen, M., Jung, W., and Turner, J., The Hypersonic Experiment ShefexAerothermodynamic Layout, Vehicle Development and First Flight Results. Proceedings of $6^{\text {th }}$ International Symposium on Launcher Technologies 'Flight Environment Control for Future and Operational Launchers', Munich, Germany. 2005.

4. Bugel, M., Reynier, P., and Smith, A., Survey of European and Major ISC Facilities for Supporting Mars and Sample Return Mission Aerothermodynamics and Tests Required for Thermal Protection System and Dynamic Stability. International Journal of Aerospace Engineering, 2011. 2011: pp. 18.

5. Leonardo, S. and Iain, B., Numerical Simulation of Weakly Ionized Hypersonic Flow for Reentry Configurations. AIAA Paper 2006-3773.

6. Casseau, V., Espinoza, D.E., Scanlon, T.J., and Brown, R.E., A Two-Temperature Open-Source CFD Model for Hypersonic Reacting Flows, Part Two: Multi-Dimensional Analysis. Aerospace, 2016. 3(4): p. 45.

7. Lani, A., An Object Oriented and High-performance Platform for Areothermodynamics Simulations. PhD Thesis, Von Karman Institute for Fluid Dynamics, 2009.

8. Ait-Ali-Yahia, D. and Habashi, W.G., Finite Element Adaptive Method for Hypersonic Thermochemical Nonequilibrium Flows. AIAA Journal, 1997. 35(8): pp. 1294-1302

9. $\quad$ Kirk, B.S., Stogner, R.H., Bauman, P.T., and Oliver, T.A., Modeling Hypersonic Entry with the Fully-Implicit NavierStokes (FIN-S) Stabilized Finite Element Flow Solver. Computers \& Fluids, 2014. 92(0): pp. 281-292.

10. Selmin, V., The Node-centred Finite Volume Approach: Bridge Between Finite Differences and Finite Elements. Computer Methods in Applied Mechanics and Engineering, 1993. 102(1): pp. 107-138.

11. Selmin, V. and Formaggia, L., Simulation of Hypersonic Flows on Unstructured Grids. International Journal for Numerical Methods in Engineering, 1992. 34(2): pp. 569-606.

12. Lee, J.H., Basic Governing Equations for the Flight Regimes of Aeroassisted Orbital Transfer Vehicles. AIAA Paper 1984-1729.

13. Gnoffo, P.A., Gupta, R.N., and Shinn, J.L., Conservation Equations and Physical Models for Hypersonic Air Flows in Thermal and Chemical Nonequilibrium. NASA TM-2867, 1989.

14. Park, C., Nonequilibrium Hypersonic Aerothermodynamics. 1989, John Wiley \& Sons, Inc.: New York.

15. Roe, P.L., Approximate Riemann Solvers, Parameter Vectors, and Difference Schemes. Journal of Computational Physics, 1981. 43(2): pp. 357-372.

16. Van Leer, B., Towards the Ultimate Conservative Difference Scheme. V. A Second-order Sequel to Godunov's Method. Journal of Computational Physics, 1979. 32(1): pp. 101-136.

17. Millikan, R.C. and White, D.R., Systematics of Vibrational Relaxation. The Journal of Chemical Physics, 1963. 39(12): pp. 3209-3213.

18. Venkatakrishnan, V., Convergence to Steady State Solutions of the Euler Equations on Unstructured Grids with Limiters. Journal of Computational Physics, 1995. 118(1): pp. 120-130.

19. Svehla, R.A., Transport Coefficients for the NASA Lewis Chemical Equilibrium Program. NASA TM-4647, 1995.

20. Casseau, V., Scanlon, T.J., and Brown, R.E., Development of a Two-temperature Open-source CFD Model for Hypersonic Reacting Flows. AIAA Paper 2015-3637. 